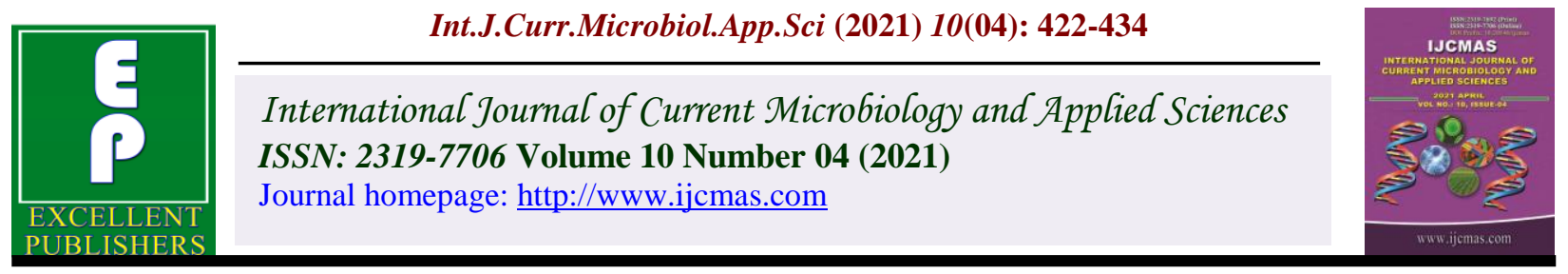

Original Research Article

https://doi.org/10.20546/ijcmas.2021.1004.045

\title{
Effect of 'P' Concentration on Biomass, Cell Integrity and Biochemical Constituents of Lyngbya sp. BDU 90901
}

\author{
Veena Dalavai, Paramageetham Chinthala* and Prasada Babu Gundala \\ Department of Microbiology, Sri Venkateswara University, Tirupati- 517502, \\ Andhra Pradesh, India \\ *Corresponding author
}

A B S T R A C T

Keywords

Cyanobacteria, phosphate limitation, growth, pigments and protein content

Article Info

Accepted:

12 March 2021

Available Online:

10 April 2021
Today world has facing the major problem of global energy demand due to continuous utilization of finite nature of fossil fuels coupled with the hike in oil prices and increase in world's population. Several researchers focused interest on microalgae and made great efforts on biofuel production to substitute fossil fuels. This paper deals with the phosphate limitation on growth, biomass, cell integrity and biochemical constituents of marine cyanobacterium, Lyngbya sp. BDU 90901. Lyngbya sp. BDU 90901 exhibited optimum growth $(\mu=0.63)$, fresh biomass $(3.3 .7 \mathrm{~g} / \mathrm{l})$, dry biomass $(0.67 \mathrm{~g} / \mathrm{l})$, chlorophyll-a $(9.3 \mu \mathrm{g} / \mathrm{ml})$, carotenoids $(2.0 \mu \mathrm{g} / \mathrm{ml})$, carbohydrate $(600 \mu \mathrm{g} / \mathrm{ml})$ at $25^{\text {th }}$ day and protein $(25 \mu \mathrm{g} / \mathrm{ml})$ at $20^{\text {th }}$ day of incubation.

\section{Introduction}

Phosphorous is a vital component essential for growth and development of algae as it constitutes nearly $1 \%$ of algal dry biomass (Hu, 2004; Borchardt and Azad, 1968). Phosphorous is a another limiting nutrient for microalgae in many natural habitats (Larned, 1998). Microalgae are good alternative, renewable source for biofuel production and are responsible for global carbon fixation (Field, et al., 1998). In natural habitats, cyanobacterial growth and metabolism is greatly affected by the important components such as phosphorous, nitrogen, carbon and iron (Eng-wilmont and Martin, 1977). Phosphorous plays an important role in the cyanobacterial growth and nitrogen fixation process (Paerl, 1990; Yandigeri et al., 2010). Several cyanobacteria can able to accumulate nearly $60 \%$ of carbohydrates under stress conditions by applying nutrient limitation strategy (Markou et al., 2012; GonzalezFernandez 2012). The biofuel production from algal biomass is environmentally and economically renewable. Microalgae uses solar energy, carbon dioxide from atmosphere and produce biomass with high lipid content 
that can be used for production of biodiesel (John, et al., 2011; Spiertz and Ewert, 2009; Petrou and Pappis, 2009). According to Beherens and kyle (1996) and Hsieh and Wu (2009), the growth and metabolism of microalgae can be altered using nutrient limitation concept.

The growth of microalgae depends not only on the availability of macronutrients but also on the micronutrients supply (Sunda, 1997). Microalgae protein can be used for human consumption, animal feed, using genetic manipulation, biofuel production can be achieved (Becker, 2007; Potvin and Zhang, 2010; Williams, and Laurens, 2010). The present paper aims at the study of phosphate effect on growth, biomass, cell integrity, photosynthetic pigments, carbohydrate and protein content of marine cyanobacterium, Lyngbya sp. BDU 90901.

\section{Materials and Methods}

A marine cyanobacterium, Lyngbya sp. BDU 90901 was obtained from National Facility for Marine Cyanobacteria (NFMC), Bharathidasan University, Tiruchirappalli, Tamil Nadu, collected from marine waters. ASN-III media was used for the marine cyanobacterial culture (Table 1). In ASN-III media, to study the effect of Phosphorus (P), Dipotassium hydrogen phosphate was used at various concentrations (Table 2). Lyngbya sp. BDU 90901 cultures were incubated at $24^{\circ} \mathrm{C} \pm 2{ }^{\circ} \mathrm{C}$ under a light intensity of 2000 lux with $16 \mathrm{~h}$ light / 8h dark conditions in ASN-III media.

A $30 \mathrm{ml}$ of optimally grown (O.D $\left.{ }_{750} 1.5\right)$ cyanobacterial culture was inoculated into 270 $\mathrm{ml}$ of basal medium in 500ml Erlenmayer flask and incubated for 30 days. At every 5 days of interval the parameters such as biomass, cell integrity and biochemical studies were conducted.

\section{Specific growth rate measurement}

Specific growth rate $(\mu)$ of Lyngbya sp. BDU 90901 was calculated according to Kratz and Myers (1955) using the following formula,

Specific growth rate; $\mathrm{K}^{\prime}$

$2.303 \log \mathrm{N}_{\mathrm{t}}-\log \mathrm{N}_{0}$

$$
\left(\mathrm{T}_{\mathrm{t}}-\mathrm{T}_{0}\right)
$$

Where $N_{t}$ is the final optical density at $750 \mathrm{~nm}$ at time $\mathrm{T}_{\mathrm{t}}$ (exponential phase), $\mathrm{N}_{0}$ is the initial optical density at $750 \mathrm{~nm}$ at time $\mathrm{T}_{0}$.

Divisions per day; Div.day ${ }^{-1}=K^{\prime} / \log 2$

Generation time; Gen’t $=1 /$ Div $^{\prime}$ day $^{-1}$

\section{Biomass estimation}

Lyngbya sp. BDU 90901 biomass estimation under various ' $\mathrm{P}$ ' limitations were carried out according to Kliphuis et al., (2012) for every 5 days until 30 days. Cultures were concentrated by centrifugation at $12,000 \mathrm{rpm}$ for 10 minutes. The cell pellet was washed with sterile water to remove traces of medium by repeated centrifugation at $12,000 \mathrm{rpm}$ for 10 minutes. The fresh weight was recorded and expressed in $\mathrm{g} / \mathrm{l}$. Then the fresh biomass was placed in hot air oven at $60^{\circ} \mathrm{C}$ for 30 minutes to obtain dry weight of the biomass.

\section{Confocal laser scanning microscopy}

The confocal laser scanning microscopic analysis of various nitrate stress conditions was carried out by using fluorescent stain, acridine orange. Cyanobacterial cell cultures grown under different phosphate conditions were centrifuged at $7000 \mathrm{rpm}$ for 10 minutes. Supernatant was removed and PBS buffer was added to the pellet. The culture was centrifuged at $7000 \mathrm{rpm}$ for 10 minutes and PBS buffer was removed. $0.2 \mathrm{ml}$ of acridine 
orange (concentration) staining solution was added to the cells and incubated at $37^{\circ} \mathrm{C}$ for 30 minutes under dark condition. After incubation, the staining solution was removed and cells were washed by PBS buffer for thrice. Then, the sample was placed on the microscopic slide and cover slip was placed over it. The microscopic slide was observed under Confocal Laser Microscope LSM 880 Carl Zeiss at excitation wavelength at 420-460 $\mathrm{nm}$ and emission wavelength at $630-650 \mathrm{~nm}$.

\section{Estimation of photosynthetic pigments}

Under various ' $\mathrm{P}$ ' concentrations, photosynthetic pigments namely chlorophylla, carotenoids and phycobiliproteins were estimated for Lyngbya sp. BDU 90901.

In Lyngbya sp. BDU 90901, chlorophyll-a and carotenoids estimations were carried out according to Sinetova et al., (2012) under different phosphate limitation conditions. 1.0 $\mathrm{ml}$ of each homogenized cyanobacterial culture was taken in centrifuge tube. Cells were centrifuged at $15,000 \mathrm{rpm}$ at laboratory temperature for 7 minutes and thoroughly supernatant was discarded. $1.0 \mathrm{ml}$ of methanol was added to the sample was homogenized by vortexing. The samples were incubated at $4{ }^{\circ} \mathrm{C}$ for 7 minutes and then centrifuged at 15,000 rpm for 7 minutes then the resultant pellet was bluish and purple in color. Replicates were maintained for calculations of averages and standard errors. The pigments concentrations were measured by using methanol as blank by spectrophotometer at $420 \mathrm{~nm}, 665 \mathrm{~nm}$ and 720 nm.

The concentration of chlorophyll a content was calculated by using the formula,

Chl $-\mathrm{a}[\mu \mathrm{g} / \mathrm{ml}]=12.9447\left(\mathrm{~A}_{665}-\mathrm{A}_{720}\right)$

Carotenoids $[\mu \mathrm{g} / \mathrm{ml}]=\left[1000\left(\mathrm{~A}_{470}-\mathrm{A}_{720}\right)-\right.$ $2.86(\mathrm{Chl}-\mathrm{a}[\mu \mathrm{g} / \mathrm{ml}] / 221$

\section{Estimation of Carbohydrates}

Lyngbya sp. BDU 90901 carbohydrates were estimated according to Gerhardt et al., (1994). $1.0 \mathrm{ml}$ of homogenized cyanobacterial suspension was taken in a test tube. $4.0 \mathrm{ml}$ of anthrone reagent and covered with foil and mixed thoroughly. The tubes were boiled for 10 minutes in water bath. The mixture was cooled to room temperature and absorbance was measured at $620 \mathrm{~nm}$ in UV-Visible spectrophotometer. The total carbohydrates content was calculated using a standard curve established with a glucose solution.

\section{Estimation of protein}

The protein content of Lyngbya sp. BDU 90901 was estimated according to Lowry et al., (1951) and Herbert et al., (1971). $1.0 \mathrm{ml}$ of homogenized cyanobacterial suspension was taken in test tubes. $0.5 \mathrm{ml}$ of reagent $\mathrm{A}$ was added and boiled for 10 minutes and cooled under running tap water. $2.5 \mathrm{ml}$ of reagent $\mathrm{B}$ was added in each tube and incubated at room temperature for 10 minutes. $0.5 \mathrm{ml}$ of regent $\mathrm{C}$ was added and the tubes were kept at room temperature for 15 minutes. The intensity of blue color was read as absorbance at $650 \mathrm{~nm}$ using appropriate blank. The measurement of protein content was done using standard calibration curve prepared from bovine serum albumin.

\section{Results and Discussion}

Lyngbya sp. BDU 90901cultured in ASN-III media prepared with various phosphate concentrations and then incubated at $24^{\circ} \mathrm{C} \pm 2^{\circ} \mathrm{C}$ under a light intensity of 2000 lux with $16 \mathrm{~h}$ light / $8 \mathrm{~h}$ dark conditions (Fig. 1).

Acridine orange staining revealed high red fluorescence indicate high cell integrity in presence of phosphorus for Lyngbya sp. BDU 90901 cyanobacterial cultures (Fig. 3). 
Lyngbya sp. BDU 90901 showed maximum growth rate $(\mu=0.63)$ at $25^{\text {th }}$ day of incubation when compared to other phosphate limitations such as $0 \mathrm{P}, 1 / 2 \mathrm{P}, 1 \mathrm{P}$ and $2 \mathrm{P}$ conditions (Table 3). Lyngbya sp. BDU 90901 produced maximum fresh biomass of $3.37 \mathrm{~g} / 1$ and dry biomass of $0.67 \mathrm{~g} / 1$ at $25^{\text {th }}$ day than $0 \mathrm{P}, 1 / 2 \mathrm{P}, 1 \mathrm{P}$ and 2P conditions (Fig. 2).

Lyngbya sp. BDU 90901 biochemical constituents such as photosynthetic pigments, protein and carbohydrates were measured under various 'P' concentrations. Chlorophylla content was high in Lyngbya sp. BDU 90901 grown $1 \frac{1}{2} \mathrm{P}$ phosphorus concentration at $25^{\text {th }}$ day (Table 4). A high carotenoid content was found at $1 \frac{1}{2} \mathrm{P}$ phosphorus concentration at $25^{\text {th }}$ day for Lyngbya sp. BDU 90901 (Table 5). In Lyngbya sp. BDU 90901, maximum carbohydrate content was found at $1 \frac{1}{2} \mathrm{P}$ phosphorus concentration at $25^{\text {th }}$ day (Table 6). A high protein content was found at $1 \frac{1}{2} \mathrm{P}$ phosphorus concentration at $25^{\text {th }}$ day for Lyngbya sp. BDU 90901 (Table 7).

Cyanobacteria cultures under nutrient limitation strategy, produce valuable industrial important products (Lan and Liao, 2011; Anfelt et al., 2015; Hirokawa, 2015; Kusakabe, 2013; Wang, 2013). The most significant contribution of this study is to determine the effect of phosphorous on marine cyanobacterium, Lyngbya sp. BDU 90901 by employing phosphate limitation strategy. Lyngbya sp. BDU 90901, a filamentous, marine, unbranched, non-heterocystous without akinetes and presence of an outer thick sheath. In the study of phosphate limitation, various growth parameters like biomass, chlorophyll-a, carotenoids, total carbohydrate and protein contents were analysed in each and every limitation. measured. In our study, the phosphate limitation on the growth and metabolism of Lyngbya sp. BDU 90901 was studied. The optimum growth of marine cyanobacteria,
Lyngbya sp. BDU 90901. was observed at $11 / 2 \mathrm{P}$ concentration of phosphate in phosphate limitation when compared to other phosphate concentrations such as $0 \mathrm{P}, 1 / 2 \mathrm{P}, 1 \mathrm{P}$ and $2 \mathrm{P}$. Phosphorus greatly influences acetylene reduction or nitrogen fixation rate in algae (Dilworth, 1966; Schollhorn and Burris, 1966; Stewart et al., 1967, 1968; Stewart et al., 1970). The growth of Synechoccus sp. was inhibited during low concentration of di potassium hydrogen phosphate (Ernst et al., 2005). Kuffner and Paul (2001) observed that with increase in phosphorous concentration, Lyngbya mujuscula growth also increased. Cyanobacteria can able to grow in high phosphate and low nitrate conditions (Sellner,1997). According to Spencer and Lembi (1981), O'Neal et al., (1985) and Dodds and Gudder (1992), algal growth is greatly influenced by ' $\mathrm{P}$ ' limitation. Dillon and Rigler (1974), Jones and Bachmann (1976), Schindler (1978) and Prepas and Trew (1983) determined correlation between the total phosphorus and algal biomass. Kozlowska- Szerenos et al., (2004) observed increase in carbonic anhydrase activities in Chlorella when cultured in absence of phosphorous. According to Lapointe (1989), Larned (1998), Schaffelke and Klumpp (1998) and Russ and Mc Cook (1999), the growth of many algae was greatly influenced by the availability of phosphorous. In the present work, Lyngbya sp. BDU 90901showed low fresh and dry biomass content in phosphorus depletion condition, increase in phosphate concentrations in the media, the biomass also increased and further increasing results in decreased in biomass content. The confocal laser scanning microscopic analysis of Lyngbya sp. BDU 90901 revealed that the phosphate enriched rich cultures exhibit high flourosecnce than phosphate deprived conditions. The chlorophyll-a content, carotenoids, phycobilisomes, total carbohydrate and protein content of Lyngbya sp. 
Table.1 Composition of ASN-III medium, [Rippka et al., 1979]

\begin{tabular}{|c|c|}
\hline Name of the chemical & Quantity (g/l) \\
\hline $\mathrm{NaCl}$ & 25 \\
\hline $\mathrm{MgCl}_{2} \cdot 6 \mathrm{H}_{2} \mathrm{O}$ & 2.0 \\
\hline $\mathrm{KCl}$ & 0.5 \\
\hline $\mathrm{NaNO}_{3}$ & 0.75 \\
\hline $\mathrm{K}_{2} \mathrm{HPO}_{4} \cdot 3 \mathrm{H}_{2} \mathrm{O}$ & 0.02 \\
\hline $\mathrm{MgSO}_{4} \cdot \mathrm{H}_{2} \mathrm{O}$ & 3.5 \\
\hline $\mathrm{CaCl}_{2} .2 \mathrm{H}_{2} \mathrm{O}$ & 0.5 \\
\hline Citric acid & 0.003 \\
\hline Ferric ammonium citrate & 0.003 \\
\hline EDTA (disodium salt) & 0.00055 \\
\hline $\mathrm{NaNO}_{3}$ & 0.02 \\
\hline Trace metal mix & $1 \mathrm{ml}$ \\
\hline Distilled water & $1000 \mathrm{ml}$ \\
\hline pH & 7.5 \\
\hline Trace metal mix Name of the chemical & Quantity (g/l) \\
\hline $\mathrm{H}_{3} \mathrm{BO}_{3}$ & 2.86 \\
\hline $\mathrm{MnCl}_{2} \cdot \mathbf{4} \mathrm{H}_{2} \mathrm{O}$ & 1.81 \\
\hline $\mathrm{ZnSO}_{4} .7 \mathrm{H}_{2} \mathrm{O}$ & 0.222 \\
\hline $\mathrm{Na}_{2} \mathrm{MoO} 4.2 \mathrm{H}_{2} \mathrm{O}$ & 0.39 \\
\hline $\mathrm{CuSO}_{4} .5 \mathrm{H}_{2} \mathrm{O}$ & 0.079 \\
\hline $\mathrm{Co}\left(\mathrm{NO}_{3}\right)_{2} \cdot 6 \mathrm{H}_{2} \mathrm{O}$ & 0.0494 \\
\hline
\end{tabular}

Table.2 Preparation of various phosphate $(\mathrm{P})$ limitation media

\begin{tabular}{|c|c|}
\hline $\begin{array}{c}\text { Name of the } \\
\text { phosphate } \\
\text { limitation }\end{array}$ & $\begin{array}{c}\text { Concentration of } \\
\text { Dipotassium } \\
\text { hydrogen } \\
\text { phosphate }(\mathbf{g} / \mathbf{l})\end{array}$ \\
\hline $\mathbf{0 P}$ & $0 \mathrm{~g} / \mathrm{l}$ \\
\hline $\mathbf{1} / 2 \mathbf{P}$ & $0.02 \mathrm{~g} / 1$ \\
\hline $\mathbf{1 P}$ & $0.04 \mathrm{~g} / 1$ \\
\hline $\mathbf{1} 1 \mathbf{P} \mathbf{P}$ & $0.06 \mathrm{~g} / 1$ \\
\hline $\mathbf{2 P}$ & $0.08 \mathrm{~g} / 1$ \\
\hline
\end{tabular}


Table.3 Specific growth rate, Divisions per day and Generation time of Lyngbya sp. BDU 90901 under different phosphate limitation.

\begin{tabular}{|c|c|c|c|c|c|c|c|c|c|c|c|c|c|c|c|}
\hline \multirow{2}{*}{$\begin{array}{c}\text { Days of } \\
\text { incubation }\end{array}$} & \multicolumn{5}{|c|}{ Specific growth $(\mu)$} & \multicolumn{5}{|c|}{ Divisions/day } & \multicolumn{5}{|c|}{ Generation time (td) } \\
\hline & OP & $1 / 2 \mathrm{P}$ & $1 \mathrm{P}$ & $1 \frac{1}{2} \mathrm{P}$ & $2 \mathrm{P}$ & $\mathrm{OP}$ & $1 / 2 \mathrm{P}$ & $1 \mathrm{P}$ & $11 / 2 \mathrm{P}$ & $2 \mathrm{P}$ & $0 \mathrm{~N}$ & $1 / 2 \mathrm{P}$ & $1 \mathrm{P}$ & $1 \frac{1}{2} 2 \mathrm{P}$ & $2 \mathrm{P}$ \\
\hline $\mathbf{0}$ & 0.02 & 0.02 & 0.02 & & 0.02 & 076 & 0.076 & .070 & $0.0 / 0$ & 0.076 & 13.15 & 15.15 & 13.15 & 13.15 & 13.15 \\
\hline 5 & 045 & .085 & 0223 & ( & 0.1 & 149 & 0.282 & 0.531 & 0.40 & 0.332 & 6.71 & 3.54 & 1.88 & 1.35 & 3.01 \\
\hline 10 & 075 & 0.134 & 0.367 & 0.26 & 0.184 & 0.249 & 0.445 & 0.863 & 1.21 & 0.611 & 4.01 & 2.24 & 1.15 & 0.78 & 1.63 \\
\hline 15 & 125 & 0.186 & 43 & 0.42 & 0.24 & 0.415 & 0.617 & 1.395 & 1.428 & 0.797 & 2.40 & 1.62 & 0.71 & 0.70 & 1.25 \\
\hline 20 & 0.195 & 0.25 & 0.49 & 0.59 & 0.36 & 0.647 & 0.830 & 1.960 & 1.627 & 1.196 & 1.54 & 1.20 & 0.51 & 0.61 & 0.83 \\
\hline 25 & 0.2 & .298 & 5 & 0.63 & 0.43 & 0.664 & 0.990 & 2.093 & 1.661 & 1.428 & 1.50 & 1.01 & 0.47 & 0.60 & 0.70 \\
\hline 30 & 0.040 & 0.14 & 0.24 & 0.38 & 0.34 & 0.301 & 0.465 & 1.262 & 0.797 & 1.129 & 3.32 & 2.15 & 0.79 & 1.25 & 0.88 \\
\hline
\end{tabular}

Table.4 Effect of phosphate on chlorophyll-a concentration $(\mu \mathrm{g} / \mathrm{ml})$ of Lyngbya sp. BDU 90901

\begin{tabular}{|c|c|c|c|c|c|c|c|}
\hline \multirow[t]{2}{*}{$\begin{array}{l}\text { Phosphate } \\
\text { Concentration }\end{array}$} & \multicolumn{7}{|c|}{$\begin{array}{c}\text { Chlorophyll-a concentration }(\mu \mathrm{g} / \mathrm{ml}) \text { of Lyngbya sp. BDU } 90901 \text { grown under } \\
\text { phosphate concentrations }\end{array}$} \\
\hline & $0^{\text {th }}$ day & $5^{\text {th }}$ day & $10^{\text {th }}$ day & $15^{\text {th }}$ day & $20^{\text {th }}$ day & $25^{\text {th }}$ day & $30^{\text {th }}$ day \\
\hline OP & $0.001 \pm 0.0001$ & $0.19 \pm 0.005$ & $0.72 \pm 0.01$ & $1.28 \pm 0.07$ & $3.09 \pm 0.003$ & $3.5 \pm 0.009$ & $3.0 \pm 0.002$ \\
\hline $1 / 2 \mathbf{P}$ & $0.014 \pm 0.0005$ & $0.12 \pm 0.004$ & $0.43 \pm 0.01$ & $2.57 \pm 0.06$ & $3.99 \pm 0.03$ & $3.98 \pm 0.05$ & $3.01 \pm 0.02$ \\
\hline 1P & $0.017 \pm 0.0003$ & $0.45 \pm 0.1$ & $0.82 \pm 0.12$ & $1.41 \pm 0.13$ & $3.87 \pm 0.14$ & $6.45 \pm 0.3$ & $5.4 \pm 0.21$ \\
\hline $11 / 2 \mathbf{P}$ & $0.01 \pm 0.004$ & $0.74 \pm 0.01$ & $2.96 \pm 0.19$ & $3.44 \pm 0.23$ & $5.96 \pm 0.16$ & $9.3 \pm 0.25$ & $8.5 \pm 0.24$ \\
\hline $2 \mathbf{P}$ & $0.01 \pm 0.03$ & $0.49 \pm 0.01$ & $2.3 \pm 0.17$ & $3.4 \pm 0.16$ & $4.77 \pm 0.24$ & $4.64 \pm 0.24$ & $3.23 \pm 0.015$ \\
\hline
\end{tabular}

Values are mean of replicates \pm standard deviation.

Table.5 Effect of phosphate on carotenoids concentration $(\mu \mathrm{g} / \mathrm{ml})$ concentration of Lyngbya sp. BDU 90901

\begin{tabular}{|c|c|c|c|c|c|c|c|}
\hline \multirow[t]{2}{*}{$\begin{array}{c}\text { Phosphate } \\
\text { Concentration }\end{array}$} & \multicolumn{7}{|c|}{$\begin{array}{l}\text { Carotenoids concentration }(\mu \mathrm{g} / \mathrm{ml}) \text { of Lyngbya sp. BDU } 90901 \text { grown under phosphate } \\
\text { concentrations }\end{array}$} \\
\hline & $0^{\text {th }}$ day & $5^{\text {th }}$ day & $10^{\text {th }}$ day & $15^{\text {th }}$ day & $20^{\text {th }}$ day & $25^{\text {th }}$ day & $30^{\text {th }}$ day \\
\hline $\mathbf{O P}$ & $0.004 \pm 0.0007$ & $0.06 \pm 0.0006$ & $0.21 \pm 0.008$ & $0.38 \pm 0.004$ & $0.65 \pm 0.007$ & $1.1 \pm 0.004$ & $1 \pm 0.009$ \\
\hline $1 / 2 \mathbf{P}$ & $0.014 \pm 0.0001$ & $0.07 \pm 0.002$ & $0.2 \pm 0.001$ & $0.32 \pm 0.006$ & $0.57 \pm 0.008$ & $1.17 \pm 0.007$ & $1.2 \pm 0.008$ \\
\hline 1P & $0.017 \pm 0.001$ & $0.09 \pm 0.0005$ & $0.24 \pm 0.001$ & $0.42 \pm 0.006$ & $0.53 \pm 0.002$ & $1.31 \pm 0.007$ & $0.87 \pm 0.003$ \\
\hline $1 / 2 \mathbf{P}$ & $0.01 \pm 0.001$ & $0.08 \pm 0.007$ & $0.28 \pm 0.001$ & $0.59 \pm 0.007$ & $0.73 \pm 0.005$ & $2.0 \pm 0.006$ & $0.98 \pm 0.004$ \\
\hline $2 \mathbf{P}$ & $0.01 \pm 0.001$ & $0.09 \pm 0.006$ & $0.32 \pm 0.002$ & $0.79 \pm 0.001$ & $1.15 \pm 0.001$ & $1.51 \pm 0.001$ & $0.95 \pm 0.001$ \\
\hline
\end{tabular}


Fig.1 Growth cultures of Lyngbya sp. BDU 90901 at different 'P' limitation
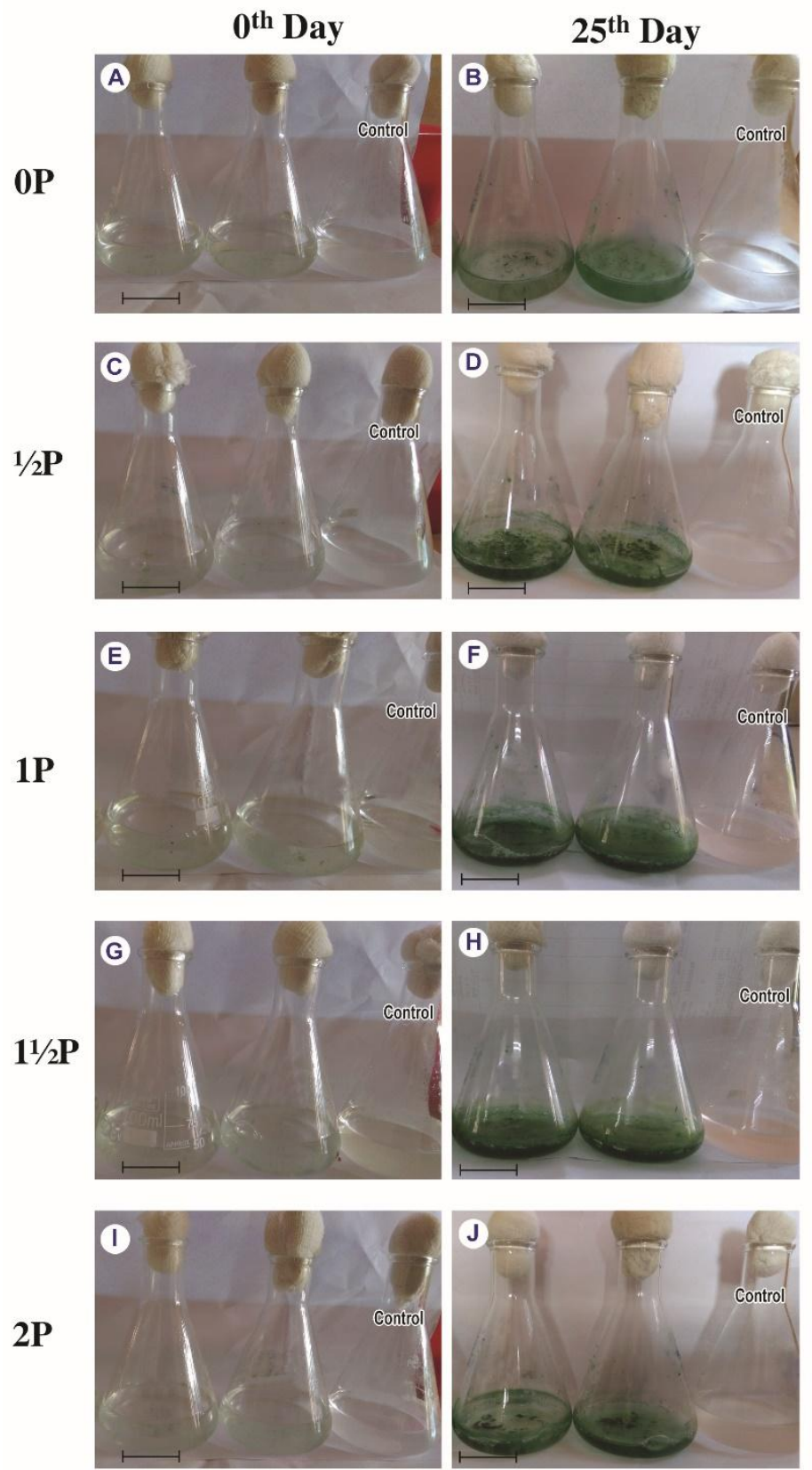

$1 \mathrm{~cm}$ bar indicates $2 \mathrm{~cm}$.

$\mathrm{A}=$ Lyngbya $\mathrm{sp}$. BDU 90901 growth cultures in OP at $0^{\text {th }}$ day $\mathrm{B}=$ Lyngbya $\mathrm{sp}$. BDU 90901 growth cultures in $0 \mathrm{P}$ at $25^{\text {th }}$ day $\mathrm{C}=$ Lyngbya $\mathrm{sp}$. BDU 90901 growth cultures in $1 / 2 \mathrm{P}$ at $0^{\text {th }}$ day $\mathrm{D}=$ Lyngbya sp. BDU 90901 growth cultures in $1 / 2 \mathrm{P}$ at $25^{\text {th }}$ day $\mathrm{E}=$ Lyngbya $\mathrm{sp} . \mathrm{BDU} 90901$ growth cultures in $1 \mathrm{P}$ at $0^{\text {th }}$ day $\mathrm{F}=$ Lyngbya sp. BDU 90901 growth cultures in $1 \mathrm{P}$ at $25^{\text {th }}$ day $\mathrm{G}=$ Lyngbya sp. BDU 90901 growth cultures in $1 \frac{1}{2} \mathrm{P}$ at $0^{\text {th }}$ day $\mathrm{H}=$ Lyngbya sp. BDU 90901 growth cultures in $1 \frac{1}{2} \mathrm{P}$ at $25^{\text {th }}$ day $\mathrm{I}=$ Lyngbya $\mathrm{sp}$. BDU 90901 growth cultures in $2 \mathrm{P}$ at $0^{\text {th }}$ day $\mathrm{J}=$ Lyngbya sp. BDU 90901 growth cultures in $2 \mathrm{P}$ at $25^{\text {th }}$ day 
Fig.2 Biomass estimation of Lyngbya sp. BDU 90901 at different 'P' limitation
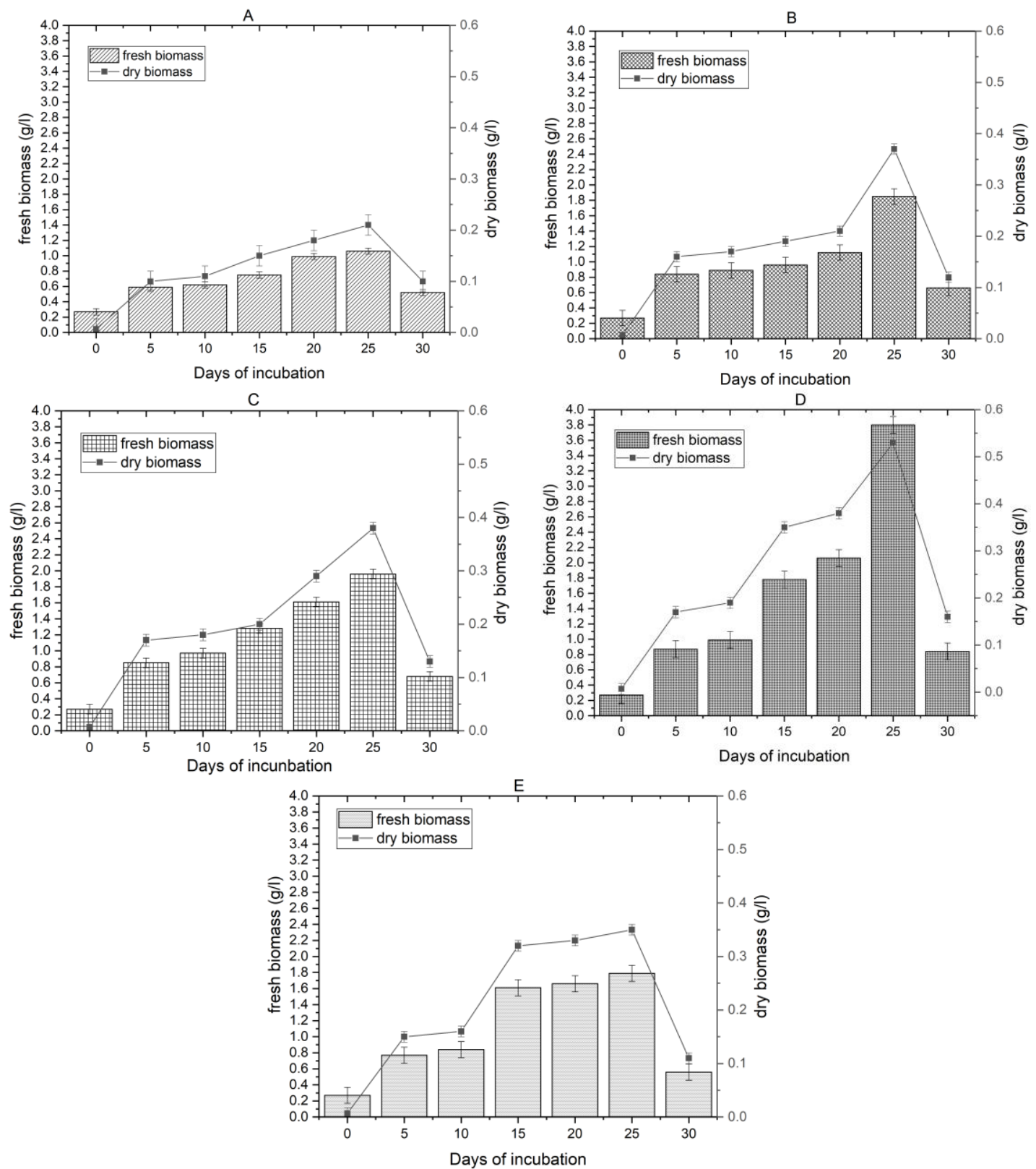

$\mathrm{A}=$ Lyngbya $\mathrm{sp}$. BDU 90901 biomass grown in 0P phosphorus concentration

$\mathrm{B}=$ Lyngbya $\mathrm{sp}$. BDU 90901 biomass grown in $1 / 2 \mathrm{P}$ phosphorus concentration

$\mathrm{C}=$ Lyngbya $\mathrm{sp}$. BDU 90901 biomass grown in 1P phosphorus concentration

$\mathrm{D}=$ Lyngbya sp. BDU 90901 biomass grown in $1 \frac{1}{2} \mathrm{P}$ phosphorus concentration

$\mathrm{E}=$ Lyngbya $\mathrm{sp}$. BDU 90901 biomass grown in 2P phosphorus concentration 
Fig.3 Confocal laser scanning microscopic images of Lyngbya sp. BDU 90901 at different 'P' limitation
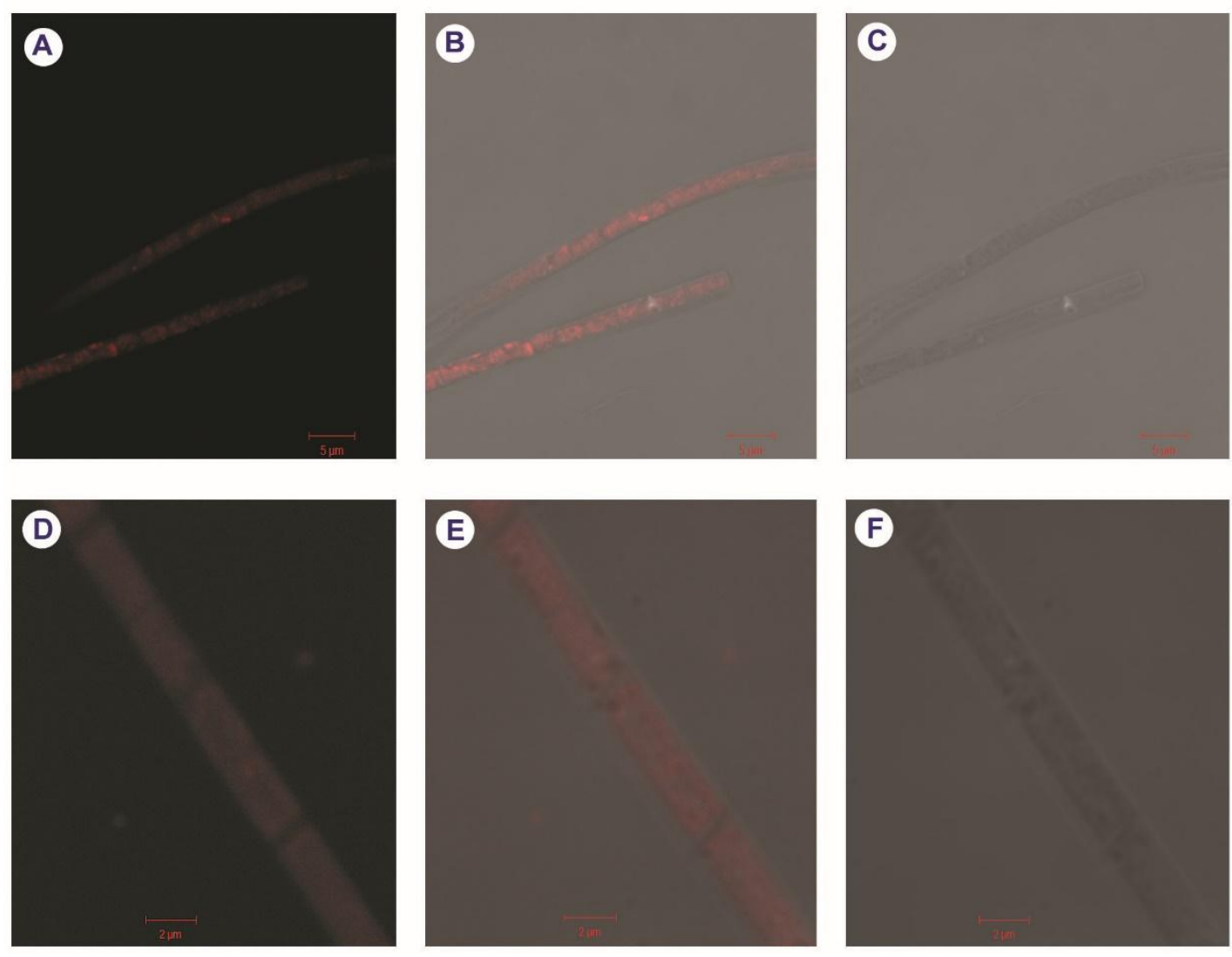
$\mathrm{A}=$ Fluorescence image of Lyngbya sp. BDU 90901 grown in ASN-III medium without phosphorus
$\mathrm{B}=$ Merged image of fluorescence and bright field of Lyngbya sp. BDU 90901 grown in ASN-III medium without phosphorus
$\mathrm{C}=$ Bright field image Lyngbya sp. BDU 90901 grown in ASN-III medium without phosphorus
$\mathrm{D}=$ Fluorescence image of Lyngbya sp. BDU 90901 grown in ASN-III medium with phosphorus
$\mathrm{E}=$ Merged image of fluorescence and bright field of Lyngbya sp. BDU 90901 grown in ASN-III medium with phosphorus
$\mathrm{F} \quad=$ Bright field image Lyngbya sp. BDU 90901 grown in ASN-III medium with phosphorus

Table.6 Total carbohydrate content ( $\mu \mathrm{g} / \mathrm{ml})$ of Lyngbya sp. BDU 90901 at different 'P' concentrations

\begin{tabular}{|c|c|c|c|c|c|c|c|}
\hline \multirow[t]{2}{*}{$\begin{array}{c}\text { Phosphate } \\
\text { Concentration }\end{array}$} & \multicolumn{7}{|c|}{$\begin{array}{c}\text { Total carbohydrates content }\left(\begin{array}{c}\mu \mathrm{g} / \mathrm{ml}) \text { of Lyngbya sp. BDU } 90901 \text { at different 'P' } \\
\text { concentrations }\end{array}\right.\end{array}$} \\
\hline & $0^{\text {th }}$ day & $5^{\text {th }}$ day & $10^{\text {th }}$ day & $15^{\text {th }}$ day & $20^{\text {th }}$ day & $25^{\text {th }}$ day & $30^{\text {th }}$ day \\
\hline OP & $5.2 \pm 0.07$ & $21 \pm 0.14$ & $54 \pm 0.16$ & $76 \pm 0.19$ & $80 \pm 0.28$ & $90 \pm 0.34$ & $40 \pm 0.35$ \\
\hline $1 / 2 \mathbf{P}$ & $5.2 \pm 0.06$ & $65.7 \pm 0.12$ & $100=$ & $125 \pm 0.17$ & $126.6 \pm 0.29$ & $100 \pm 0.26$ & $87.54 \pm 0.38$ \\
\hline 1P & $5.2 \pm 0.02$ & $70.5 \pm 0.18$ & 20 & 21 & 25 & $200 \pm$ & $175 \pm 0.22$ \\
\hline $1 / 2 P$ & $5.2 \pm 0.03$ & $110 \pm 0.19$ & $453 \pm 0.24$ & $484 \pm 0.20$ & $532 \pm 0.11$ & $600 \pm 0.29$ & $194 \pm 0.05$ \\
\hline $2 \mathbf{P}$ & $5.2 \pm 0.04$ & $65 \pm 0.14$ & $106.5 \pm 0.28$ & $150 \pm 0.36$ & $250 \pm 0.58$ & $98.3 \pm 0.33$ & $79.43 \pm 0.16$ \\
\hline
\end{tabular}

Values are mean of replicates \pm standard deviation. 
Table.7 Protein content $(\mu \mathrm{g} / \mathrm{ml})$ of Lyngbya sp. BDU 90901 at different 'P' concentrations

\begin{tabular}{|c|c|c|c|c|c|c|c|}
\hline $\begin{array}{c}\text { Phosphate } \\
\text { Concentration }\end{array}$ & \multicolumn{6}{|c|}{ Protein content $(\boldsymbol{\mu g} / \mathbf{m l}) \begin{array}{c}\text { of Lyngbya sp. BDU 90901 at different 'P' } \\
\text { concentrations }\end{array}$} \\
\cline { 2 - 8 } & $\mathbf{0}^{\text {th }}$ day & $\mathbf{5}^{\text {th }}$ day & $\mathbf{1 0}^{\text {th }}$ day & $\mathbf{1 5}^{\text {th }}$ day & $\begin{array}{c}\mathbf{2 0}^{\text {th }} \\
\text { day }\end{array}$ & $\begin{array}{c}\mathbf{2 5}^{\text {th }} \\
\text { day }\end{array}$ & $\begin{array}{c}\mathbf{3 0}^{\text {th }} \\
\text { day }\end{array}$ \\
\hline $\mathbf{0 P}$ & $10 \pm 0.015$ & $10.42 \pm 0.1$ & $11.9 \pm 0.13$ & $13 \pm 0.16$ & $14 \pm 0.24$ & $18 \pm 0.23$ & $12 \pm 0.18$ \\
\hline $\mathbf{1} / \mathbf{P}$ & $10 \pm 0.08$ & $13.4 \pm 0.2$ & $16 \pm 0.18$ & $18 \pm 0.33$ & $22 \pm 0.37$ & $15 \pm 0.31$ & $13 \pm 0.31$ \\
\hline $\mathbf{1 P}$ & $10 \pm 0.09$ & $16.7 \pm 0.31$ & $17 \pm 0.11$ & $19 \pm 0.21$ & $21 \pm 0.38$ & $23 \pm 0.33$ & $18 \pm 0.28$ \\
\hline $\mathbf{1} 1 / 2 \mathbf{P}$ & $10 \pm 0.09$ & $15.31 \pm 0.15$ & $18.6 \pm 0.24$ & $22.8 \pm 0.28$ & $25 \pm 0.32$ & $16 \pm 0.34$ & $14 \pm 0.23$ \\
\hline $\mathbf{2 P}$ & $10 \pm 0.07$ & $11.49 \pm 0.16$ & $14 \pm 0.39$ & $18 \pm 0.32$ & $20 \pm 0.31$ & $22 \pm 0.37$ & $21 \pm 0.31$ \\
\hline
\end{tabular}

Values are mean of replicates \pm standard deviation.

BDU 90901 was low during phosphate starved conditions and this result correlates with the reports of Yandigeri et al., (2010) that total chlorophlly-a content, total carbohydrate, total soluble protein were very low at phosphate deprived conditions in case of Anabena variabilis and Westiellopsis prolifica. According to Dean et al., (2008) and Sigee et al., (2007), Chlamydomonas was greatly affected by the phosphate stress than Scenedesmus, as cyanobacteria showed high carbohydrate content in phosphate limitation conditions. Several reports stated that in algae during phosphorous depletion, the decline in chlorophyll-a and protein content as well as increase in carbohydrate content (Kilham et al., 1997; Healey, 1982; Healey and Hendzel, 1979). During phosphorous deprivation condition, decrease in phycobilisomes was observed by Collier and Grossman (1992). Marine-algae contains phosphatases which plays an important role in recycling and availability of phosphorous in the sea (Hoppe, 2003). Several reports states that cyanobacteria can able to grow in low phosphate availability conditions by hydrolysis of organic phosphorous (Grainger et al., 1989; Mahasneh et al., 1990; Whitton et al., 1990, 1991, 1998; Palenik and Dyhrman, 1998; Scanlan and Wilson, 1999; Stihl et al., 2001). In the present work, under phosphate stress marine cyanobacterium, Lyngbya sp. BDU 90901 showed highest growth rate, fresh biomass, dry biomass, chlorophyll-a, carotenoids, total sugar and protein content in $1 \frac{1}{2} \mathrm{P}$ phosphate concentration. The confocal laser scanning microscopic observation also revealed that the marine cyanobacterial cultures grown in phosphate enriched media showed high florescence than in phosphate starvation condition. From the results the carbohydrates enriched marine cyanobacterial biomass was further used for bioethanol production.

\section{Statistical analysis}

The obtained results were analyzed with the statistical data analysis using one - way ANOVA, SPSS 20 version, Advanced MSExcel.

\section{Acknowledgement}

The authors would like to acknowledge Department of Science and TechnologyINSPIRE program, New Delhi, India for providing financial support to the research work.

\section{References}

Anfelt, J., Kaczmarzyk, D., Shabestary, K., Renberg, B., Rockberg, J., Nielsen, J., Uhlen, M., and Hudson, E. P. (2015). Genetic and nutrient modulation of 
acetyl-CoA levels in Synechocystis for n-butanol production. Microbial Cell Factories, 14, 167.

Becker, E. W. 2007. Micro-algae as a source of protein. Biotechnol. Adv. 25, 207-210.

Beherans, P. W. Kyle, D. J. Microalgae as a source of fatty acids. J Food Lipids 1996; 3:259-72.

Borchardt, L. J. A., Azad, H. S. Biological extraction of nutrients. J. Water Pollut. Control Fed. 1968, 40, 1739-1754.

Collier J L and Grossman A R (1992) Chlorosis induced by nutrient deprivation in Synechococcus sp. Strain PCC 7942: Not all bleaching is the same. $\mathrm{J}$ Bacteriol 174: 4718-4726.

Dean, A. P., Nicholson, J. M., Sigee D. C. Impact of phosphorous quota and growth phase on carbon allocation in Chlamydomonas reinhardtii: an FTIR microspectroscopy study. Eur J Phycol 2008; 43:345-54.

Dillon, P. J., and Rigler, F. H. (1974). The phosphorus-chlorophyll relationship in lakes. Limnology and Oceanography, 19, 767-773.

DiLworth, M. J. (1966). Acetylene reduction by nitrogen-fixing preparations from Clastridium pasteurianum. Biochimica et Biophysica Acta, 111, 285-294.

Dodds, W. K., and Gudder, D. A. (1992). The ecology of Cladophora. Journal of Phycology, 28, 415-427.

Eng-Wilmot, D. L., and Martin, D. F. 1977. Growth response of the marine bluegreen alga, Gomphosphaeria aponina, to inorganic nutrients and significance to management of Florida red tide. Microbios. 1977;19(77-78):167-79.

Ernst A, Deicher M, Herman PM \& Wollenzien UIA (2005) Nitrate and phosphate affect cultivability of cyanobacteria from environments with low nutrient levels. Appl Environ Microbiol 71: 3379-3383.

Field, C.B., Behrenfeld, M.J., Randerson, J. T., Falkowski, P. Primary production of the biosphere: integrating terrestrial and oceanic components. Science 1998. 281:237-40.
Gerhardt, P., Murray, R. G. E., Wood, W. A., and Krieg, N. R. (1994). Methods for General and Molecular Bacteriology, ASM, Washington DC.p-518.

Gonzalez-Fernandez, C., and Ballesteros, M. (2012). Linking microalgae and cyanobacteria culture conditions and key-enzymes for carbohydrate accumulation. Biotechnology Advances. 30, 1655-1661.

Healey, F. P. Phosphate. Biol. Cyanobacteria 1982, 19, 105-124.

Healey, F. P., Hendzel, L. L. Indicators of phosphorous and nitrogen deficiency in five algae in culture. J. Fish. Board Can. 1979, 36, 1364-1369.

Hirokawa, Y., Suzuki, I., and Hanai, T. (2015). Optimization of isopropanol production by engineered cyanobacteria with a synthetic metabolic pathway. Journal of Bioscience and Bioengineering,119, 585-590.

Hoppe, H.G. 2003. Phosphatase activity in the sea. Hydrobiology, 493:187-200.

Hsieh, C.H., Wu, W.T. Cultivation of microalgae for oil production with a cultivation strategy of urea limitation. Bioresour Technol 2009; 100:3921-6.

$\mathrm{Hu}, \mathrm{Q}$. Environmental effects on cell composition. In Handbook of Microalgal Culture: Biotechnology and Applied Phycology; Richmond, A., Ed.; Blackwell: Oxford, UK, 2004; pp-83-93.

John, R. P., Anisha, G. S., Nampoothiri K. M., Pandey, A. Microalgal and macroalgal biomass: a renewable source for bioethanol. Bioresour Technol 2011; 102:186-93.

Jones, J. R., and Bachmann, R. W. (1976). Prediction of phosphorus and chlorophyll levels in lakes. Journal of Water Pollution Control Federation, 48, 2176-2182.

Kilham, S., Kreeger, D., Gouldon, C., Lynn, S. Effects of nutrient limitation on biochemical constituents of Ankistrodesmus falcatus. Freshw. Biol. 1997, 38,591-596. 
Kliphuis, A. M. J., Klok, A. J., Martens, D. E., Lamers, P. P., Akedo, M., and Wijffels, R H. (2012). Metabolic modeling of Chlamydomonas reinhardtii: energy requirements for photoautotrophic growth and maintenance. Journal of Applied Phycology, 24, 253-266.

Kozolwska-Szerenos, B., Bialuk, I., Maleszewski, S. Enhancement of photosynthetic $\mathrm{O}_{2}$ evolution in Chlorella vulgaris under high light and increased $\mathrm{CO}_{2}$ concentration as a sign of acclimation to phosphate deficiency. Plant Physiol Biochem 2004; 42:403-9.

Kratz, W. A., Myers, J. (1955). Nutrition and growth of several blue green algae. Amm. J. Bot. 42, 282-287.

Kuffner IB, Paul VJ (2001) Effects of nitrate, phosphate and iron on the growth of macroalgae and benthic cyanobacteria from Cocos Lagoon, Guam. Mar Ecol Prog Ser 222:63-72.

Kusakabe, T., Tatsuke, T., Tsuruno, K., Hirokawa, Y., Atsumi, S., Liao, J. C., and Hanai T. (2013). Engineering a synthetic pathway in cyanobacteria for isopropanol production directly from carbon dioxide and light. Metabolic Engineeing, 20, 101-108.

Lan, E. I., and Liao, J. C. (2011). Metabolic engineering of cyanobacteria for 1butanol production from carbon dioxide. Metabolic Engineering, 13, 353-363.

Larned, S. Nitrogen-versus phosphorous-limited growth and sources of nutrients for coral reef macroalgae. Mar. Biol. $1998,132,409-421$.

Lowry, O. H., Rosebrough, N. J., Farr, A. L., and Randall, R. J. (1951). Protein measurement with the Folin phenol reagent. Journal of Biological Chemistry, 193, 265-275.

Markou, G., Angelidaki, I., Georgakakis, D. Microalgal carbohydrates: An overview of the factors influencing carbohydrates production and of main bioconversion technologies for production of biofuels. Appl. Microbiol. Biotechnol. 2012, 96, 631-645.
O’Neal, S. W., Lembi, C. A., and Spencer, D. L. (1985). Productivity of the filamentous alga Pithophora oedogonia (Chlorophyta) in Surrey Lake, Indiana. Journal of Phycology, 21, 562-569.

Paerl, H. W. (1990). Physiological ecology and regulation of $\mathrm{N}_{2}$ fixation in natural waters. Adv Microbiol Ecol 11:305-344.

Petrou, E. C. Pappis, C. P. Biofuels: a survey on pros and cons. Energy Fuels 2009; 23:1055-66.

Potvin, G., and Zhang, Z. 2010. Strategies for high level recombinant protein expression in transgenic microalgae: a review. Biotechnol Adv. 28. 910-918.

Prepas, E. E., and Trew, D. O. (1983). Evaluation of the phosphorus chlorophyll relationship for lakes of the Precambrian Shield in western Canada. Canadian Journal of Fisheries and Aquatic Sciences, 40, 27-35.

Rippka, R., Deruelles, J., Waterbury, J. B., Herdman, M., and Stainer, R. Y. (1979). Generic assignment, strain histories and properties of pure cultures of cyanobacteria. Journal of General Microbiology, 111, 1-61.

Schindler, D. W. (1978). Factors regulating phytoplankton production and standing crop in the world's freshwaters. Limnology and Oceanography, 23, 478486.

Schollhorn, R., and Burris, R. H. (1966). Study of intermediates in nitrogen fixation. Federation of American Societies for Experimental Biology, 25, 10.

Sellner, K. G. (1997). Physiology, ecology, and toxic properties of marine cyanobacterial blooms. Limnol. Oceanogr., 42: 10891104.

Sigee, D. C., Bahrami, F., Estrada, B., Webster, R. E., Dean, A. P. The influence of phosphorous availability on carbon allocation and P quota in Scendesmus subspicatus: a synchrotron-based FTIR analysis. Phycology 2007; 46:583-92.

Sinetova, M. A., Kupriyanova, E. V., Markelova, A. G., Allakhverdiev, S. I., and Pronina, N. A. (2012). Identification 
and functional role of the carbonic anhydrase Cah3 in thylakoid membranes of pyrenoid of Chlamydomonas reinhardtii. Biochimica et Biophysica Acta, 1817, 1248-1255.

Spencer, D. L., and Lembi C A. (1981). Factors regulating the spatial distribution of the filamentous alga Pithophora oedogonia (Chlorophyceae) in an Indiana lake. Journal of Phycology. 17,168-173.

Spiertz, J. H. J., Ewert, F. Crop production and resource use to meet the growing demand for food, feed and fuel: opportunities and constraints. NJASWageningen J Life Sci 2009; 56:281300.

Stewart, W. D. P., Fitzgerald, G. F., and Burris, R. H. (1968). Acetylene reduction by nitrogen fixing blue-green algae. Archieves in Microbiology, 62, 336-348.

Stewart, W. D. P., Fitzgerald, G. P., and Burris, R. H. (1967). In situ studies on $\mathrm{N}_{2}$ fixation using the acetylene reduction technique. Proceedings of National Academy of Sciences of United States of America, 58, 2071-2078.
Stewart, W. D. P., Fitzgerald, G. P., and Burris, R. H. (1970). Acetylene reduction assay for determination of phosphorus availability in Wisconsin lakes. Proceedings of National Academy of Sciences of United States of America, 66, 1104-1111.

Sunda, W. G., Huntsman S. A. Interrelated influence of iron, light and cell size on marine phytoplankton growth. Nature 1997; 390:389-92.

Wang, W., Liu, X., and Lu, X. (2013). Engineering cyanobacteria to improve photosynthetic production of alka(e)nes. Biotechnology for Biofuel, 6, 69.

Williams, P. J. B., Laurens, L. M. 2010. Microalgae as biodiesel and biomass feedstocks: reviews and analysis of the biochemistry, energetics and economics. Energy Environ. Sci 3. 554-590.

Yandegeri, M. S., Yadav, A. K., Meena, K. K., Pabbi, S. 2010. Effect of mineral phosphates on growth and nitrogen fixation of diazotrophic cyanobacteria Anabaena variabilis and Westiellopsis prolifica. Antonie van Leeuwenhoek 97(3):297-306.

\section{How to cite this article:}

Veena Dalavai, Paramageetham Chinthala and Prasada Babu Gundala. 2021. Effect of 'P' Concentration on Biomass, Cell Integrity and Biochemical Constituents of Lyngbya sp. BDU 90901. Int.J.Curr.Microbiol.App.Sci. 10(04): 422-434.

doi: https://doi.org/10.20546/ijcmas.2021.1004.045 